\title{
Review on Smart Health Care Monitoring Systems
}

\author{
Vasishth V. Katre, Dr. P. N. Chatur
}

Department of Computer Science \& Engineering, Government College of Engineering Amravati, Maharashtra, India

\section{ABSTRACT}

Article Info

Volume 7, Issue 4

Page Number: 418-422

Publication Issue :

July-August-2021

\section{Article History}

Accepted : 20 July 2021

Published : 27 July 2021
Document IoT is leading in smart health care system. Using different sensors it's possible to monitor the patients' healthcare remotely. This is unimagined and leads to a spatial longitude amalgamated with machine learning approach. Leading to smart health care, and headway in medical field. It may lead to know severe health issues ahead of time which would be tranquil to the health system. Which would benefit the hospital administration and management. This paper elucidates on the distinct sort of IoT based health care monitoring systems. The aim is to juxtapose the present health care IoT systems.

Keywords :- Internet of Things (IoT), Smart Healthcare, Health Monitoring, Machine Learning

\section{INTRODUCTION}

With an advancement in science and technology, medical science the longevity of an individual has increased substantially over the globe along with dwindle in mortality rate. After math the number of elder people has steadily increased. According to 2019s world population ageing survey there are $9 \%$ of the world's population elderly and by 2050 it is predicted to be $16 \%$. Which may lead to stress on medical aspects. IoT based Technology can deliver a sustainable amount of information regarding human application medical devices. Amalgamation of modern internet technology and internet of things (IoT) provides a great innovative scope in medical field, based on lo3w cost wireless communication. It offers continues monitoring of the health of an individual offering collection and processing of data. There exist several health monitoring systems. Different sensors can be attached to a patients or an individual's body in order to obtain the healthcare based medical data and stored at the server. Here at the server the data can be processed with machine learning aspects as well as by the doctors for diagnosis.

\section{LITERATURE SURVEY}

In paper [1] the author proposes system to maintain and observe daily behaviour of an individual via using Wearable Internet of Things (W-IoT) ,human activity recognition based on Improved Bayesian Convolution Network the doesn't propose to work over real-time data. It just maintains the individual's behaviour and stores it for observing the activity.

As IoT is expanding its roots everywhere it's also useful in E-Health care. People are getting more health conscious, different wearable devices are being used to monitor their daily activity and a kept a track

Copyright: @ the author(s), publisher and licensee Technoscience Academy. This is an open-access article distributed under the terms of the Creative Commons Attribution Non-Commercial License, which permits unrestricted non-commercial use, distribution, and reproduction in any medium, provided the original work is properly cited 
of their daily routine of an individual. The author proposes an E-Health care monitoring system that monitors the health of an individual, transmit the data to the server and stores it. SVM a Machine Learning algorithm is applied to the transmitted data in order to analyse the received data. With the machine learning approach if any abnormality is found in the data set, the prediction of the disease is done and immediate treatment is alert is sent so that treatment can be started fast. The system also keeps a record of doctor's appointments and gives an alert of an appointment, suggest food diet, stores E-health care reports. [2]

Continuous health monitoring of critically ill patient who need to be monitored minutely and with high accuracy in order to take proper decisions for doctor but it's very hard to spontaneously keep a track of every moment of the patient, in order to keep a continuous track of critical patient the author as introduced a health monitoring system using Zigbee and GSM. The system sends a SMS alert to the doctor if there's an abnormal or unpredictable action with the patient. [3]

The author as developed a system for monitoring temperature remotely, here the xbee a wireless communication is used for the transmission of data along with aurdino. The temperature of patient is monitored and saved, if any abnormality is observed the systems gives an alert to the doctor.[4]

In this paper [5] the author has used deep learning for intelligent decision making in order to determine the state of patient through the data received to the IoT cloud. Electroencephalogram (EEG) sensors are use here for acquiring accurate data from the patient. For fast processing of the data all the process is done on the cloud, cloud integration is used.

A healthcare system, which can monitor location of a critical patient in order to reach him through GPS Neo6. After locating the patient the emergency services can be made available to the patient as the doctors, ambulance can reach his location by using Google map. Here the GPS sensor GPS Neo6 is connected to the arduino, which receives the GPS signals and sends to the server. Arduino is connected to the internet through the GSM Sim800 which is a gateway to the internet. [6]

For the expedite living the authors have introduced an IoT based system which works on hardware and data analytical module i.e. cloud. A system which monitors temperature, blood pressure, location of the patient along with the fall recognition of the patient, all data of the sensor is sent to the cloud through the arduino and processed on the cloud, if any abnormality is found in the data from the sensors an alert is sent to the nearest hospital about the patient and can be treated soon.[7]

As the age profile of people is increasing day by day, there are also affected by the chronic diseases. Chronic disease patients need a spontaneous observation over different parameters. Therefore in order to monitor these patient spontaneously a wearable IoT system is introduced, for the real-time health monitoring. In this system wearable sensors are attached to body and connected to the cloud through the zigbee for communication. [8]

The author has proposed a health monitoring and room automation system that will monitor the patient's different health aspects along with alarm generation to the doctor and patents relatives if there's any abnormality seen in the sensors threshold values. It also provides reminders for the prescribed medicines. Along with this the system also controls the room and provides an automation for the ease of the patient. All the sensors are connected to the raspberry pie which transfers the data to the cloud. [9]

In this paper [10] the author has introduced a telemedicine system health care system using Internet of Things in order to monitor elderly person who 
stays alone and needs to be observed. In order to track his heart rate, pulse rate, blood flow, from this data the health can be predicted. If there is any abnormality in the data, the doctor is informed about that so that proper treatment can be given.

The author is inter-relating the diabetes patients with the chronic kidney disease and hypertension as all three diseases are inter related they are using the health data of diabetes patient and monitoring the data and correlate it with the other diseases. A huge data base is developed by tracking patients clinical information, which could be used for clinical research. Diabetes patients have a possibility of chronic kidney disease as well as hypertension so in order to predict an emergency before time this analysis is done for the safety of the patient. [11]

In this paper [12] the author has analysed different classification algorithms in order to prognosticate diabetes patient early stage, they have tried the three classification algorithms 1 decision tree 2 Support Vector Machine 3 Naïve Buyer. In which Naïve Buyer classification algorithm outperformed the highest in case of accuracy. Naïve Buyer's accuracy was $76.30 \%$. Performance evaluation was done on Precision, Accuracy, F-measure and Recall.

Iot Devices are leading technology that are making human life easy in many ways. Number of IoT devices have been introduced but there's a need of time to work on the security of these devices. The purpose of the device is to transfer data, but the data transferred should be safe and secured. Security challenges can be can be mitigated by using the cryptography and steganography techniques. This paper deals with the security of IoT devices in deferent ways, and concluded with elliptic Galois cryptography is the best way to secure the devices.

In this paper [14] the author has suggested the stenographic scene for the safety of the data transmission through the IoT devices as the information is very sensitive and confidential. This can stop data leakage during the transmission of the data in the network. Vector quantization (VQ) is used for the transmission of the data using least significant beat, along with the compressed VQ image it reduces the size of the image and at the end point an image repairer is used for reading the $\mathrm{QR}$ compressed image.

A Decoupled Block chain is used in health monitoring system with an approach of edge envisioned for safe and secured health monitoring system. Decouples block chain helps in secure transmission of the data from the sensor to the cloud through the edge node. It separate the block headers ledgers which results in reduction of block preparation and header generation time along with secure transmission of the data. [15]

\section{III.CONCLUSION}

Implementation of present work will contribute to research in the area of Environment physiology which will form the basis for recommendations and draft guidelines on how and to what extent exposures to the extreme conditions can be tolerated in a safe way with minimized health risks considering short, medium as well as long term effects. This system that would benefit in monitoring the patients who are home taking care at home rather than getting hospitalization and also predict Health Emergency condition of a Covid19 patient based on Machine Learning approach. Which would be easier and safer for the doctors and nurses to monitor a huge number of patients being anywhere at the hospital or home.

\section{REFERENCES}

[1]. Z. Zhou, H. Yu and H. Shi, "Human Activity Recognition Based on Improved Bayesian Convolution Network to Analyze Health Care Data Using Wearable IoT Device," in IEEE Access, vol. 8, pp. 86411-86418, 2020, doi: 10.1109/ACCESS.2020.2992584. 
Vasishth V. Katre et al Int. J. Sci. Res. Comput. Sci. Eng. Inf. Technol, July-August-2021, 7 (4) : 418-422

[2]. B. Godi, S. Viswanadham, A. S. Muttipati, O. P. Samantray and S. R. Gadiraju, "E-Healthcare Monitoring System using IoT with Machine Learning Approaches," 2020 International Conference on Computer Science, Engineering and Applications (ICCSEA), Gunupur, India, 2020, $\quad$ pp. 1-5, doi: 10.1109/ICCSEA49143.2020.9132937.

[3]. Purnima Puneet Singh, "Zigbee and GSM based patient health monitoring system," 2014 International Conference on Electronics and Communication Systems (ICECS), Coimbatore, India, 2014, pp. 1-5, doi: 10.1109/ECS.2014.6892762.

[4]. H. Mansor, M. H. A. Shukor, S. S. Meskam, N. Q. A. M. Rusli and N. S. Zamery, "Body temperature measurement for remote health monitoring system," 2013 IEEE International Conference on Smart Instrumentation, Measurement and Applications (ICSIMA), Kuala Lumpur, Malaysia, 2013, pp. 1-5, doi: 10.1109/ICSIMA.2013.6717956.

[5]. S. U. Amin, M. S. Hossain, G. Muhammad, M. Alhussein and M. A. Rahman, "Cognitive Smart Healthcare for Pathology Detection and Monitoring," in IEEE Access, vol. 7, pp. 1074510753, 2019, doi: 10.1109/ACCESS.2019.2891390.

[6]. P. Kanani and M. Padole, "Real-time Location Tracker for Critical Health Patient using Arduino, GPS Neo6m and GSM Sim800L in Health Care," 2020 4th International Conference on Intelligent Computing and Control Systems (ICICCS), Madurai, India, 2020, pp. 242-249, doi: 10.1109/ICICCS48265.2020.9121128.

[7]. R. Banu, A. Taranum, D. Kirti, M. Jagadeesh, K. S. Nagaranjini and N. Thejaswini, "“An Iot Enabled Smart Health Care Kit for Expedite Living"," 2019 International Conference on Intelligent Computing and Control Systems
(ICCS), Madurai, India, 2019, pp. 1200-1204, doi: 10.1109/ICCS45141.2019.9065329.

[8]. Wan, Jie \& Al-awlaqi, Munassar \& Li, MingSong \& O'Grady, Michael \& Gu, Xiang \& Wang, Jin \& Cao, Ning. (2018). Wearable IoT enabled real-time health monitoring system. EURASIP Journal on Wireless Communications and Networking. 2018. 10.1186/s13638-0181308-x.

[9]. J. Saha et al., "Advanced IOT based combined remote health monitoring, home automation and alarm system," 2018 IEEE 8th Annual Computing and Communication Workshop and Conference (CCWC), Las Vegas, NV, 2018, pp. 602-606, doi: 10.1109/CCWC.2018.8301659.

[10]. J. Liau and C. Ho, "Intelligence IoT(Internal of Things) Telemedicine Health Care Space System for the Elderly Living Alone," 2019 IEEE Eurasia Conference on Biomedical Engineering, Healthcare and Sustainability (ECBIOS), Okinawa, Japan, 2019, pp. 13-14, doi: 10.1109/ECBIOS.2019.8807821.

[11]. Nakashima, N., Noda, M., Ueki, K. et al. Recommended configuration for personal health records by standardized data item sets for diabetes mellitus and associated chronic diseases: a report from a collaborative initiative by six Japanese associations. Diabetol Int 10, 85 92 (2019). https://doi.org/10.1007/s13340-01900389-7.

[12]. Deepti Sisodia, Dilip Singh Sisodia,Prediction of Diabetes using Classification Algorithms,Procedia Computer Science,Volume 132,2018,Pages 1578-1585,ISSN 1877-0509, https://doi.org/10.1016/j.procs.2018.05.122.

[13]. M. Khari, A. K. Garg, A. H. Gandomi, R. Gupta, R. Patan and B. Balusamy, "Securing Data in Internet of Things (IoT) Using Cryptography and Steganography Techniques," in IEEE Transactions on Systems, Man, and Cybernetics: Systems, vol. 50, no. 1, pp. 73-80, Jan. 2020, doi: 10.1109/TSMC.2019.2903785. 
[14]. Huang, CT., Tsai, MY., Lin, LC. et al. VQ-based data hiding in IoT networks using two-level encoding with adaptive pixel replacements. J Supercomput 74, 4295-4314 (2018). https://doi.org/10.1007/s11227-016-1874-9.

[15]. G. S. Aujla and A. Jindal, "A Decoupled Blockchain Approach for Edge-Envisioned IoTBased Healthcare Monitoring," in IEEE Journal on Selected Areas in Communications, vol. 39, no. 2, pp. 491-499, Feb. 2021, doi: 10.1109/JSAC.2020.3020655.

\section{Cite this article as :}

Vasishth V. Katre, Dr. P. N. Chatur, "Review on Smart Health Care Monitoring Systems", International Journal of Scientific Research in Computer Science, Engineering and Information Technology (IJSRCSEIT), ISSN : 2456-3307, Volume 7, Issue 4, pp.418-422, July-August-2021. Available at doi : https://doi.org/10.32628/CSEIT2174112 Journal URL : https://ijsrcseit.com/CSEIT2174112 